\title{
Speech Disorders in Olivopontocerebellar Atrophy Correlate with Positron Emission Tomography Findings
}

\author{
Karen J. Kluin, MS, ${ }^{*}$ Sid Gilman, MD, † Dorene S. Markel, MS, † Robert A. Koeppe PhD, \\ Guy Rosenthal, BA, $\dagger$ and Larry Junck, MD $\dagger$
}

We compared the severity of ataxic and spastic dysarthria with local cerebral metabolic rates for glucose (ICMRGlc) in 30 patients with olivopontocerebellar atrophy (OPCA). Perceptual analysis was used to examine the speech disorders, and rating scales were devised to quantitate the degree of ataxia and spasticity in the speech of each patient. ICMRGlc was measured with ${ }^{18} \mathrm{~F}$-2-fluoro-2-deoxy-D-glucose and positron emission tomography (PET). PET studies revealed marked hypometabolism in the cerebellar hemispheres, cerebellar vermis, and brainstem of OPCA patients compared with 30 control subjects. With data normalized to the cerebral cortex, a significant inverse correlation was found between the severity of ataxia in speech and the ICMRGlc within the cerebellar vermis, cerebellar hemispheres, and brainstem, but not within the thalamus. No significant correlation was found between the severity of spasticity in speech and ICMRGlc in any of these structures. The findings support the view that the severity of ataxia in speech in OPCA is related to the functional activity of the cerebellum and its connections in the brainstem.

Kluin KJ, Gilman S, Markel DS, Koeppe RA, Rosenthal G, Junck L. Speech disorders in olivopontocerebellar atrophy correlate with positron emission tomography findings. Ann Neurol 1988;23:547-554

Olivopontocerebellar atrophy (OPCA) is a progressive neurological disorder characterized by neuronal degeneration in the cerebellar cortex, pons, and inferior olives [1-4]. OPCA usually begins with a disorder of gait and soon leads to dysarthria and limb ataxia. The dysarthria is mixed, involving both ataxic and spastic elements [5]. Recently, we used ${ }^{18} \mathrm{~F}$-2-fluoro-2-deoxyD-glucose $\left({ }^{18} \mathrm{~F}-\mathrm{FDG}\right)$ and positron emission tomography (PET) to study local cerebral metabolic rates for glucose (ICMRGIc) in patients with OPCA [6]. The studies demonstrated significant hypometabolism in the cerebellar hemispheres, cerebellar vermis, and brainstem in comparison with age-matched normal control subjects.

Dysarthria results from disturbances in the muscular control of the speech mechanisms due to impairment of any of the basic processes involved in the execution of speech [7-9]. Dysarthria can result from abnormalities of articulation and also from disorders of respiration, phonation, resonance, and prosody. The major types of dysarthria are classified as flaccid, spastic, ataxic, hypokinetic, hyperkinetic, and mixed [9]. These types of dysarthria have been linked to anatomical loci of disease in the nervous system.
The structures in the central nervous system (CNS) responsible for ataxic and spastic forms of dysarthria have not been identified with certainty. Although it is generally held that the cerebellum and its connections are important in ataxic dysarthria, the relationship of the severity of dysfunction in these structures to the degree of dysarthria has not been determined. Ataxic dysarthria has been attributed principally to injury of the superior cerebellar vermis [9-11], but dysarthria has been observed with bilateral cerebellar hemisphere damage $[12,13]$, with lesions of the paravermal and lateral elements of the hemispheres $[14,15]$, and with damage to the left paravermal area [15]. The importance of brainstem structures to the pathogenesis of ataxic dysarthria is unclear. Spastic dysarthria is thought to result from bilateral dysfunction of corticobulbar connections, but further details about this pathway have not been elucidated.

Darley and associates [7-9] used perceptual analysis to identify the speech characteristics of patients with the major types of dysarthria. They termed these characteristics deviant speech dimensions and studied their frequency in patients with disorders affecting relevant regions of the nervous system. In patients with ataxic
From the Departments of "Physical Medicine and Rehabilitation, †Neurology, and $¥$ Internal Medicine, The University of Michigan, Ann Arbor, MI.

Received Sep 23, 1987, and in revised form Jan 4, 1988. Accepted for publication Jan 7, 1988.
Address correspondence to Dr Gilman, Department of Neurology, The University of Michigan, 1914/0316 Taubman Health Care Center, 1500 E. Medical Center Drive, Ann Arbor, MI 48109-0316. 
dysarthria from cerebellar disease, they identified 10 deviant dimensions that were sufficiently severe to warrant inclusion in their list. In patients with spastic dysarthria associated with pseudobulbar palsy, they found 14 deviant dimensions that met their criteria for inclusion.

In this study we have correlated the deviant dimensions characterizing ataxic and spastic dysarthria with ICMRGlc in patients with OPCA. We found it necessary to modify the lists of Darley and associates [9] to separate more completely the deviant dimensions characterizing ataxic and spastic dysarthria. Our aim was to determine whether the severity of ataxic and spastic dysarthria correlated with the metabolic rates of particular brain structures considered important in the pathogenesis of these types of dysarthria. The findings revealed a strong correlation between the severity of ataxic dysarthria and ICMRGlc in the cerebellar hemispheres, cerebellar vermis, and brainstem. Preliminary findings from this study have been published [16].

\section{Methods}

We studied 30 patients with OPCA and 30 normal controls. The studies were approved by the institutional review board of the University of Michigan Medical Center and informed consent was obtained from all subjects. The patients with OPCA included 17 women and 13 men with an average duration of illness of $6 \pm 5$ years and a range from 1 to 22 years. Fourteen patients had a positive family history and 16 patients had sporadic disease. The normal control subjects had no history of neurological disease and no significant abnormalities on neurological and general physical examination. These subjects were taking no medication known to affect CNS function or to cause CNS side effects. All subjects were carefully screened for alcoholism, and no one with a clear history of alcoholism was admitted into the study. The diagnosis of OPCA was made on the basis of the history, physical examination, neurological examination, laboratory tests to exclude other diseases, and findings on computed tomography (CT) scans. None of the patients with OPCA had disorders of sensory function adequate to cause ataxia of movement. The laboratory tests included complete blood count; serum profiles of hepatic and renal function; brainstem auditory, visual, and somatosensory evoked potentials; serum levels of vitamin $E$, vitamin $B_{12}$, and folic acid; and studies of thyroid function. A search was made for an occult malignancy and included a pelvic examination in the women, a prostate examination in the men, acid phosphatase levels, guaiac tests for occult blood, and chest roentgenograms.

Normal control subjects and OPCA patients were studied in the University of Michigan Cyclotron/PET Facility while lying supine, awake, and blindfolded in a quiet room. Each subject was placed in a head holder that maintained the head immobile throughout the study. The head was aligned along the orbitomeatal line with a laser. Scans were performed 30 to 75 minutes after injection of ${ }^{18} \mathrm{~F}$-FDG. ${ }^{18} \mathrm{~F}$-FDG was synthesized by a modification of the method of Ehrenkaufer and associates [17]. Radiochemical purity was greater than $95 \%$. Five to ten millicuries were injected intravenously and PET scans were performed with a TCC PCT 4600A tomograph having an in-plane resolution of $11 \mathrm{~mm}$ full width at half maximum (FWHM) and a $z$-axis resolution of $9.5 \mathrm{~mm}$ FWHM. Five planes with 11.5 -mm center-to-center separation were imaged simultaneously. Four sets of scans were taken per patient, including two interleaved sets through lower brain levels and two interleaved sets through higher brain levels for a total of 20 slices, each separated by 5.75 $\mathrm{mm}$. Attenuation correction was calculated by the standard ellipse method, modified to account for attenuation from the head holder and skull.

Blood samples were collected from the radial artery. ICMRGlc was calculated using a three-compartment model and single scan approximation described by Phelps and associates [18] with gray matter kinetic constants derived from normal subjects [19]. Regions of interest (ROIs) were studied in the cerebellar hemispheres, vermis, brainstem, thalamus, and cerebral cortex. As described fully in a previous communication [6], data were collected from the ROIs by placing a $22 \times 11 \mathrm{~mm}$ parallelogram over each cerebellar hemisphere, an $11 \times 18 \mathrm{~mm}$ rectangle over the vermis, an $11 \times 15 \mathrm{~mm}$ rectangle over the brainstem, and an $11 \times 11$ mm square over each thalamus. Each ROI was centered over a local peak in ICMRGlc. For reference, an individual image element (pixel) was $3.75 \mathrm{~mm} \times 3.75 \mathrm{~mm}$ in size. Data were obtained from two slices containing the cerebellum and the brainstem and from one slice containing the thalamus. ROIs from the cerebellar vermis were posterior to the fourth ventricle. The brainstem ROI chiefly reflected the pons, but the mesencephalon or the medulla oblongata could have been partially represented. Data from the cerebral cortex were obtained by measuring ICMRGlc in the cortical ribbon from five consecutive slices beginning with the lowest slice containing the basal ganglia. This was accomplished using a computerized algorithm that enhanced the contrast in the images, then selected a $15-\mathrm{mm}$-wide band extending inward from the cortical rim. The mean metabolic rate was computed for each of these ROIs.

Conventional neurological examinations were performed and recorded. Evaluation of the dysarthria included oral motor assessment and perceptual speech analysis. One of the authors $(\mathrm{K} . \mathrm{J} . \mathrm{K}$.) analyzed and rated the severity of the ataxic and spastic dysarthric components from videotaped or audiotaped samples of spontaneous speech, expository speech (description of the "cookie theft" picture from the Boston Diagnostic Aphasia Examination), oral reading of the "Grandfather Passage," diadochokinetic rates, duration of sustaining the vowel sounds $a b$ and $e e$, and counting from 1 to 75 . We used the definitions of deviant speech dimensions by Darley and associates [9]. Speech samples were assessed in multiple listening sessions with each session focused upon a single deviant dimension. A quantitative rating was developed extending from 0 (unaffected) to 3 (severely affected) and assigned to each deviant speech dimension.

For quantitation of the ataxic and spastic components of speech, we modified the Mayo Clinic rating system described by Darley and associates [9]. The Mayo Clinic lists contain several deviant speech dimensions that are found in both ataxic and spastic dysarthria (imprecise consonants, excess and equal stress, distorted vowels, harsh voice, monopitch, monoloudness, slow rate) (Table 1). In addition, some of the 
Table 1. Deviant Dimensions in Ataxic and Spastic Speech According to the Mayo Clinic Lists (9)

\begin{tabular}{ll}
\hline Ataxic Speech & Spastic Speech \\
\hline Imprecise consonants & Imprecise consonants \\
Excess and equal stress & Monopitch \\
Irregular articulatory & Reduced stress \\
breakdown & Harsh voice \\
Distorted vowels & Monoloudness \\
Harsh voice & Low pitch \\
Prolonged phonemes & Slow rate \\
Prolonged intervals & Hypernasality \\
Monopitch & Strained-strangled quality \\
Monoloudness & Short phrases \\
Slow rate & Distorted vowels \\
& Pitch breaks \\
& Breathy voice-continuous \\
& Excess and equal stress \\
\hline
\end{tabular}

Table 2. Deviant Dimensions Important in Ataxic and Spastic Speech According to the University of Michigan Lists ${ }^{\mathrm{a}}$

\begin{tabular}{ll}
\hline Ataxic Speech & Spastic Speech \\
\hline Excess and equal stress (3) & Strained-strangled quality \\
Irregular articulatory & $(3)$ \\
$\quad$ breakdown (2) & Reduced stress (2) \\
Alternating loudness (2) & Harsh voice-continuous \\
Variable rate (2) & $(2)$ \\
Fluctuating pitch (2) & Slow rate (2) \\
Harsh voice-transient (1) & Low pitch (2) \\
Breathy voice-transient (1) & Imprecise phonemes (1) \\
Altered nasality-transient & Monoloudness (1) \\
(1) & Hypernasality-continuous \\
Voice tremors (1) & $(1)$ \\
Audible inspiration (1) & Monopitch (1) \\
& Prolonged phonemes and/or \\
& intervals (1) \\
\hline
\end{tabular}

${ }^{a}$ The numbers in parentheses indicate the relative weight used for each deviant dimension in determining each patient's dysarthria rating for this study.

deviant dimensions noted to occur infrequently by Darley and associates [9] have been found frequently in our experience. For ataxic speech, these include alternating loudness, variable rate, fluctuating pitch, breathy voice, altered nasality, voice tremors, and audible inspiration. For spastic speech, these include prolonged phonemes or prolonged intervals or both. Our aim in this study was to separate ataxic dysarthria from spastic dysarthria as clearly as possible to reflect our clinical experience and to make correlations of speech abnormalities with ICMRGlc in PET studies. Accordingly, we reorganized the deviant dimensions to eliminate some of the overlap between ataxic and spastic dysarthria and to include some deviant dimensions that are relegated to positions of lesser importance in the Mayo Clinic lists (Table 2). We obtained a total ataxic dysarthria rating and spastic dysarthria rating for each patient by summing the ratings of the individual deviant dimensions.

Figure 1 shows the severity of ataxia in speech plotted against the severity of spasticity in speech using the Mayo
MAYO CLINIC CLASSIFICATION

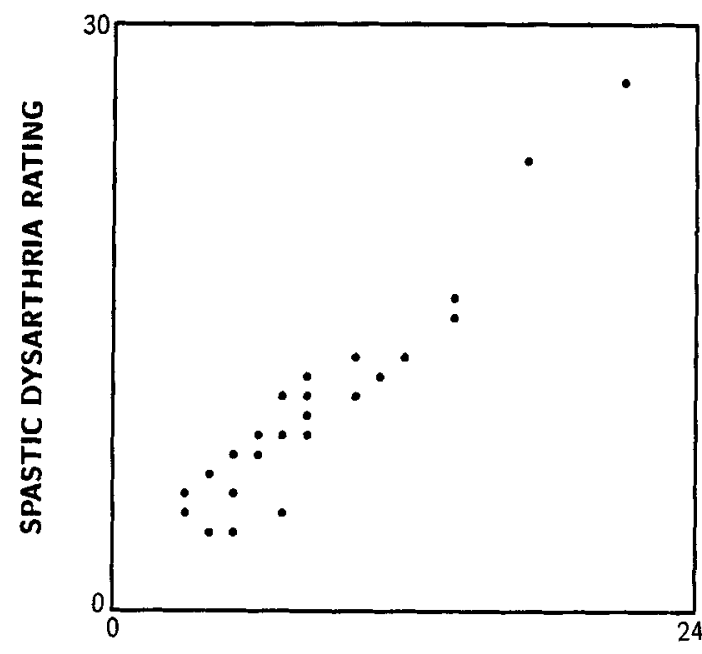

A

ATAXIC DYSARTHRIA RATING

UNIVERSITY OF MICHIGAN CLASSIFICATION

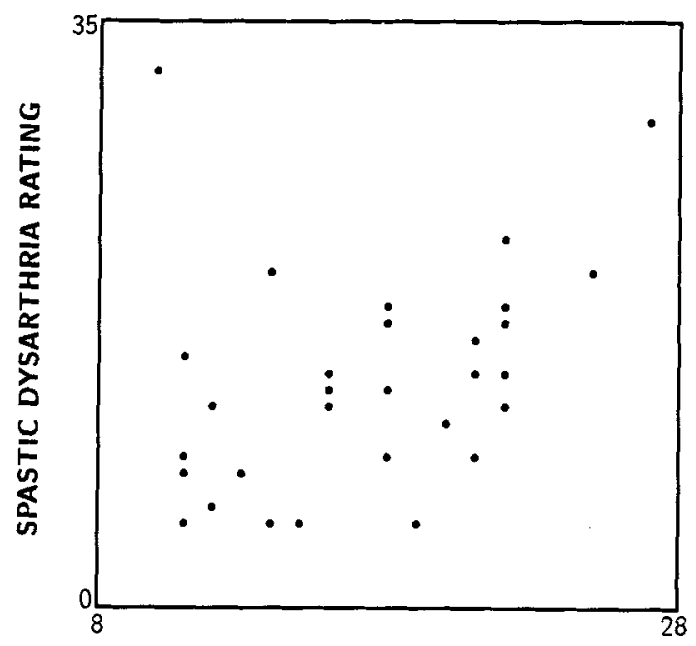

ATAXIC DYSARTHRIA RATING

B

Fig 1. Severity of ataxia in speech plotted against severity of spasticity in speecb using the Mayo Clinic lists of deviant dimensions (A) and the University of Micbigan lists (B). The strong correlation between ataxic and spastic speech in the Mayo Clinic lists results from overlapping deviant dimensions and is reduced in the University of Michigan lists.

Clinic lists (A) and our modification of these lists (B). The strong relationship between ataxic and spastic speech in the Mayo Clinic lists, largely resulting from the many overlapping deviant dimensions, became weaker in the University of Michigan modification. In the analysis of the severity of ataxic and spastic dysarthria, we applied weighting factors to enhance the impact of the deviant dimensions that we considered most characteristic of these disorders (see Table 2). Our scales ranged from 0 to 48 for ataxic dysarthria and from 


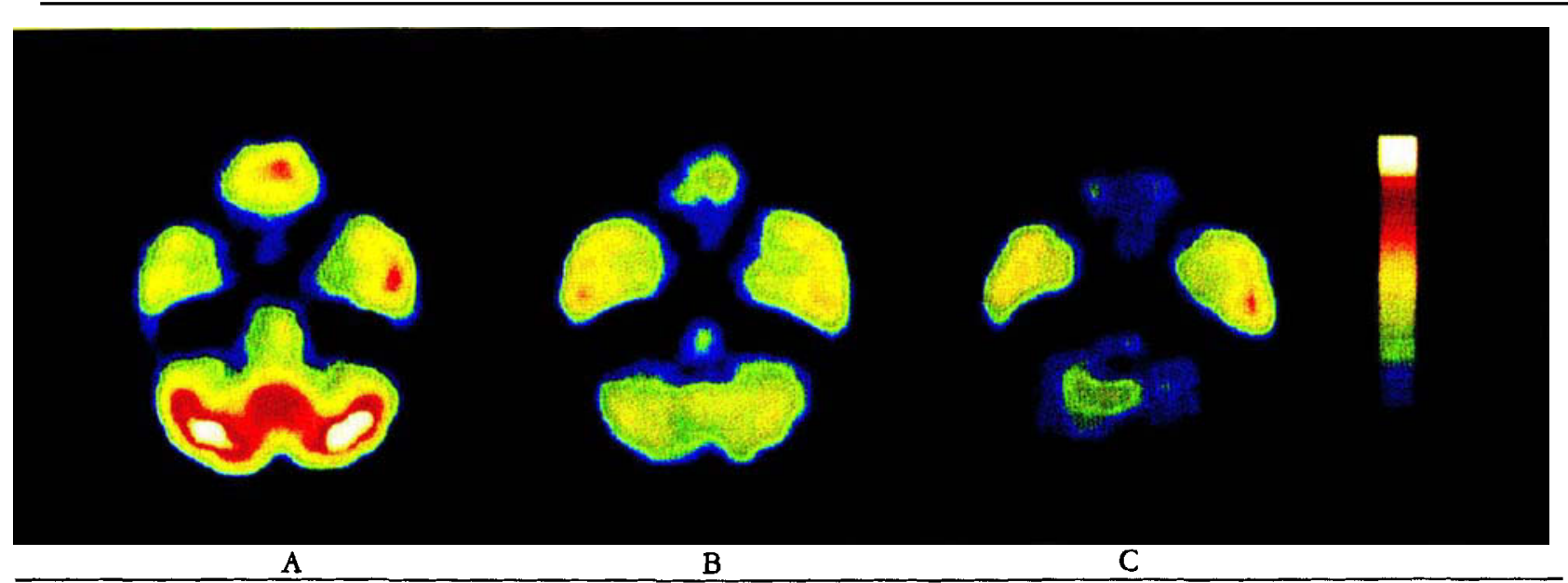

Fig 2. Positron emission tomograpby scans sbowing cerebral glucose utilization as detected with ${ }^{18}$ F-2-fluoro-2-deoxy-D-glucose. The scans show a borizontal section at the level of the cerebellum and the base of the temporal and frontal lobes. (A) Female control subject aged 64 years. Note that the glucose utilization rates are slightly bigher in the cerebellum than in the temporal lobes in this subject. Color bars indicate glucose utilization rates in $\mathrm{mg} / 100 \mathrm{gm} / \mathrm{min}$ extending from 0.0 to 7.7 . (B) Female patient aged 61 years with olivopontocerebellar atrophy. Note the moderate degree of hypometabolism in the cerebellar vermis, cerebellar bemispheres, and brainstem. This patient had a score of 12 in ataxic speech rating. Color bars indicate glucose utilization rates in $\mathrm{mg} / 100 \mathrm{gm} / \mathrm{min}$ extending from 0.0 to 7.0. (C) Female patient aged 59 years with olivopontocerebellar atropby. Note the marked degree of bypometabolism in the cerebellar vermis, cerebellar hemispheres, and brainstem. Color bars indicate glucose utilization in $\mathrm{mg} / 100 \mathrm{gm} / \mathrm{min}$ extending from 0.0 to 5.8. This patient bad a score of 21 in ataxic speech rating.

0 to 48 for spastic dysarthria. With this rating system, a score of 21 or more on either scale indicated a severe degree of dysarthria. Our decisions concerning the reorganization of the deviant dimensions characterizing ataxic and spastic dysarthria and the weighting factors were based upon our clinical experience and were made prior to any analysis of data from the PET studies.

Spearman rank correlations were used to compare metabolic data with speech characteristics. The partial correlations method was used to eliminate the effect of the relationship between ataxic and spastic speech characteristics when measuring these variables against ICMRGlc. We considered $p<0.05$ to be statistically significant.

\section{Results}

PET studies revealed marked hypometabolism in the cerebellar hemispheres, cerebellar vermis, and brainstem of OPCA patients compared with normal control subjects (Fig 2). A significant inverse correlation was found between the degree of ataxia of speech and the absolute level of ICMRGlc within the cerebellar vermis, both cerebellar hemispheres, the brainstem, and the cerebral cortex, but not within the thalamus (Table

3). A significant inverse correlation was also found between the degree of spasticity of speech and the absolute level of ICMRGlc within the cerebellar vermis, both cerebellar hemispheres, the brainstem, and the cerebral cortex, but not within the thalamus (see Table $3)$. When the metabolic data were normalized to the cerebral cortex, a significant inverse correlation was still found between the degree of ataxia of speech and the ICMRGlc within the cerebellar vermis, cerebellar hemispheres, and brainstem, but not within the thalamus (see Table 3). The normalized data revealed no significant correlation between the severity of spasticity in speech and ICMRGlc in any of the structures studied (see Table 3). Figure 3 illustrates the correlations between ICMRGlc and ataxic dysarthria ratings for the cerebellar vermis, cerebellar hemispheres, brainstem, and thalamus.

We noted that, with absolute metabolic data, both ataxic and spastic speech characteristics correlated with ICMRGlc in the cerebellum, brainstem, and cerebral cortex. This finding raised the possibility of a correlation between ataxic and spastic speech patterns. We used the partial correlations method to determine the relationship of each speech characteristic to ICMRGlc without the contribution of the other speech characteristic (Table 4). Partial correlations revealed that when the effects due to variations in the spastic speech rating were eliminated, a significant inverse correlation remained between the degree of ataxia in speech and absolute ICMRGlc in the cerebellar vermis, cerebellar hemispheres, and brainstem, but not in the thalamus or cerebral cortex (see Table 4). Absolute levels of cerebral cortical ICMRGlc no longer correlated with ataxic speech characteristics when the interactive effects of spastic speech were eliminated (compare Tables 3 and 4). Partial correlations also revealed that when the effects due to variations in the ataxic speech ratings were taken into account, a significant inverse relationship was found between the degree of spastic- 
Table 3. Correlation Coefficients between ICMRGlc and Dysarthria Ratings for Ataxic and Spastic Speech

\begin{tabular}{llcrr}
\hline & \multicolumn{2}{c}{ ICMRGlc (Absolute) $^{\mathrm{a}}$} & Spastic & \multicolumn{2}{c}{ lCMRGlc (Normalized) $^{\mathrm{b}}$} \\
\cline { 2 - 5 } Brain Region & Ataxic & $-0.45^{\mathrm{c}}$ & Ataxic & Spastic \\
\hline Cerebellar vermis & $-0.57^{\mathrm{e}}$ & $-0.51^{\mathrm{d}}$ & $-0.40^{\mathrm{c}}$ & -0.24 \\
Cerebellar hemispheres & $-0.59^{\mathrm{e}}$ & $-0.47^{\mathrm{d}}$ & $-0.48^{\mathrm{d}}$ & -0.30 \\
Brainstem & $-0.56^{\mathrm{e}}$ & -0.27 & $-0.42^{\mathrm{c}}$ & -0.10 \\
Thalamus & -0.24 & $-0.44^{\mathrm{c}}$ & -0.10 & -0.13 \\
Cerebral cortex & $-0.34^{\mathrm{c}}$ & $\ldots$ & $\ldots$ \\
\hline
\end{tabular}

${ }^{2}$ Absolute lCMRGlc in $\mathrm{mg} / 100 \mathrm{gm} / \mathrm{min}$.

blCMRGlc normalized to the cerebral cortex.

${ }^{c} p<0.05$.

$\mathrm{d}_{p}<0.005$.

${ }^{e} p<0.001$.

$1 \mathrm{CMRGlc}=$ local cerebral metabolic rate for glucose.

ity in speech and absolute ICMRGlc in the cerebellar vermis, cerebellar hemispheres, brainstem, and cerebral cortex, but not in the thalamus (see Table 4). We also used the partial correlations method on ICMRGlc data normalized to the cerebral cortex (see Table 4). Significant inverse correlations remained for ataxic dysarthria and ICMRGlc in the cerebellar vermis, cerebellar hemispheres, and brainstem, but not in the thalamus. With ICMRGlc normalized to the cerebral cortex, however, no significant correlations were found between spastic dysarthria and ICMRGlc in any of the structures studied.

\section{Discussion}

This study extends our earlier work showing a marked decrease of ICMRGlc in the cerebellar vermis, cerebellar hemispheres, and brainstem in OPCA [6]. We now show a strong inverse correlation between ICMRGlc in these structures and the severity of ataxia in speech. The correlation coefficients between absolute levels of ICMRGlc and degree of ataxia in speech were approximately equal for the left and right cerebellar hemispheres (separate data are not shown), the cerebellar vermis, and the brainstem. Reduced ICMRGlc in these structures probably resulted from diminished functional activity owing to decreased numbers of metabolically active synaptic terminals and cell bodies. Many connections have doubtlessly degenerated, since OPCA results pathologically in the progressive deterioration of numerous cellular elements in the cerebellar cortex, cerebellar nuclei, and pons $[3,4]$, and most of the patients studied showed some degree of atrophy of cerebellum and brainstem in CT scans [20].

In a previous study, we assessed the relationship of tissue atrophy, as determined by CT scans, to ICMRGlc in the cerebellum and brainstem in OPCA patients [6]. A significant relationship was found; however, several patients had minimal or no atrophy and substantially reduced ICMRGlc. The findings suggested that tissue atrophy did not fully account for the finding of hypometabolism. Currently, we cannot determine with certainty to what extent the decreased lCMRGlc values reflected decreased metabolism per gram of viable tissue and to what extent they represented decreased tissue mass with partial volume averaging.

In this study, a significant inverse correlation was found between the absolute level of ICMRGlc in the cerebral cortex and the degree of ataxia in speech, although this correlation was weaker than the correlations for the cerebellum and brainstem. This finding was unexpected, since ataxia of speech is thought to stem from disease of the cerebellum and its connections $\{3,7,9-15]$ and not from disease of the cerebral cortex. Other unexpected findings were the significant inverse correlations between the absolute levels of ICMRGlc and the severity of spasticity in speech for the cerebellar vermis, cerebellar hemispheres, and brainstem. Spasticity of speech is thought to result from disease of the corticobulbar projections [9] and not from disease of the cerebellum.

The reason for the unexpected findings described above concerns the interactive effects of ataxic and spastic characteristics. The Mayo Clinic list of deviant dimensions in ataxic speech was compiled by Darley and associates [9] by listening to the speech of patients with diseases of the cerebellum and its connections. Individual deviant speech dimensions were identified and given a severity rating in each patient. The resulting list indicates the important deviant dimensions in ataxic speech disorders. The same procedure was followed to obtain the Mayo Clinic list of deviant dimensions in spastic speech except that the patients selected had disease of the corticobulbar connections. Clinical descriptions and diagnoses of the patients studied have not been published, making it difficult to know the relative purity of the cases. Some of the deviant di- 


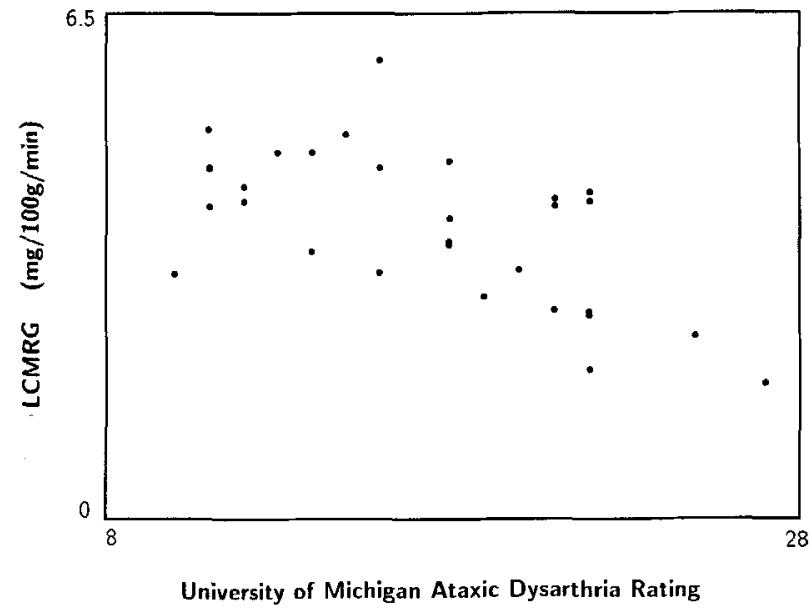

A

BRAINSTEM

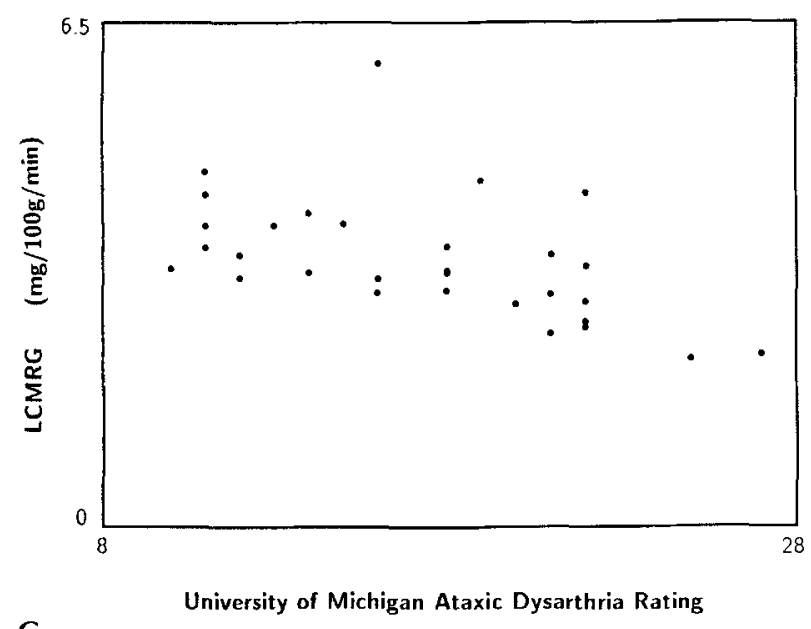

Fig 3. Graphs of local cerebral metabolic rate for glucose (lCMRGlc) in $\mathrm{mg} / 100 \mathrm{gm} / \mathrm{min}$ plotted against severity of ataxic dysartbria for the cerebellar vermis $(A)$, the average of the left and right cerebellar bemispheres $(B)$, the brainstem $(C)$, and the thalamus (D). There is a significant inverse correlation between the degree of ataxia in speech and the level of ICMRGlc within the cerebellar vermis, cerebellar hemispheres, and brainstem, but not within the thalamus. There is no difference in the results between the left and right cerebellar bemispberes.

mensions described as occurring infrequently in ataxic dysarthria by Darley and associates [9] occur frequently in our experience. Moreover, our findings differ from those of Darley and associates [9] concerning the deviant dimensions that are most characteristic of ataxic speech. We find the most important deviant dimensions to be excess and equalized stress patterns, irregular articulatory breakdown, alternating loudness, variable rate, and fluctuating pitch. In spastic speech, we find the most characteristic deviant dimensions to

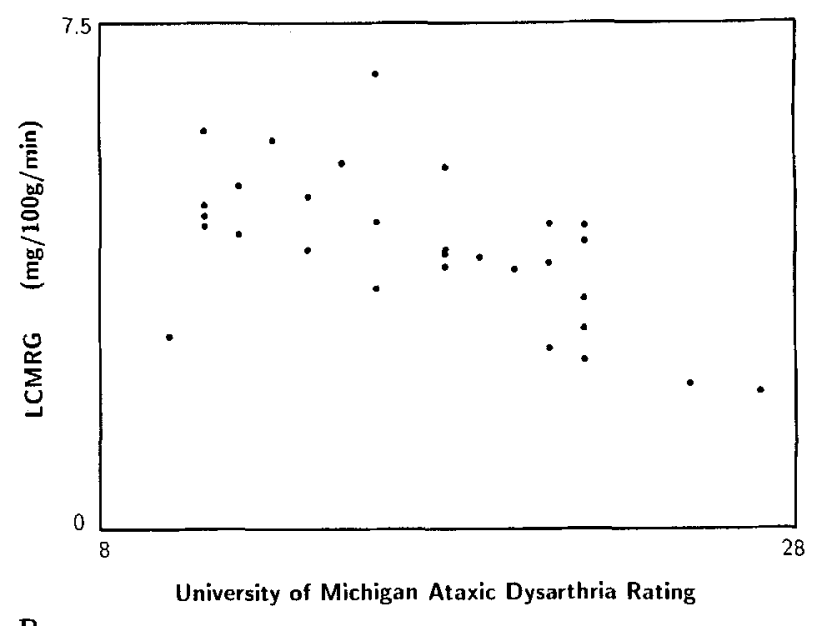

B

THALAMUS

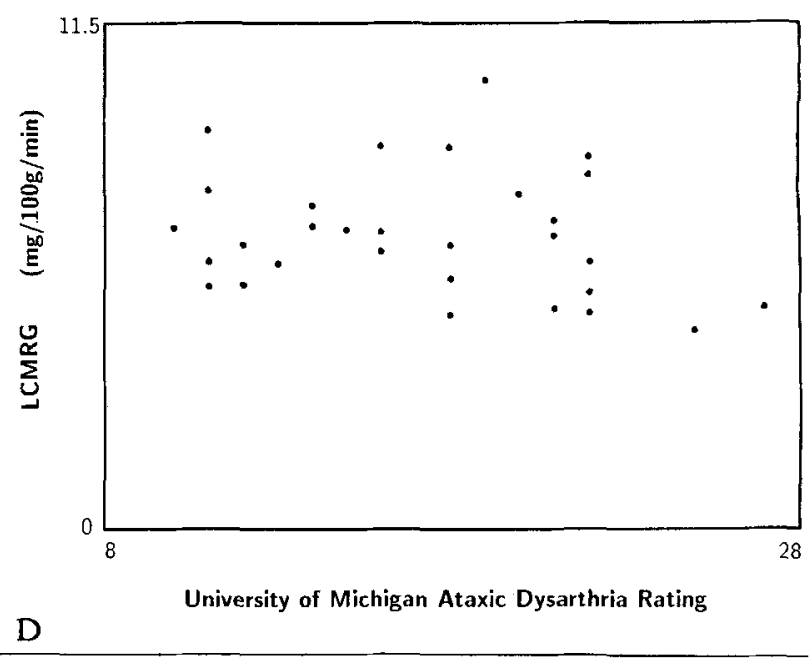

be strained-strangled quality, reduced stress, harsh voice-continuous, slow rate, and low pitch.

To analyze the speech data and make correlations with PET studies, we needed to reformulate the Mayo Clinic list of deviant dimensions. This was necessary because of the overlap of deviant dimensions between ataxic and spastic speech in the Mayo Clinic lists and because of differences between our clinical experience and that of Darley and associates [9]. We used weighting factors to enhance the importance of the deviant speech dimensions that were most responsible for the type of dysarthria found in this clinical population. Analysis by this approach separated ataxic from spastic speech characteristics fairly effectively, but a significant relationship remained between the two. As noted above, significant inverse correlations were found for both spastic and ataxic speech when compared with absolute ICMRGlc in the cerebellar vermis, cerebellar hemispheres, brainstem, and cerebral cortex. A clue to 
Table 4. Correlations between ICMRGle and Dysarthria Ratings for Ataxic and Spastic Speech as Determined by the Partial Correlations Metbod ${ }^{\text {a }}$

\begin{tabular}{|c|c|c|c|c|}
\hline \multirow[b]{2}{*}{ Brain Region } & \multicolumn{2}{|c|}{ ICMRGlc (Absolute) ${ }^{\mathrm{b}}$} & \multicolumn{2}{|c|}{ ICMRGIc (Normalized) ${ }^{\mathrm{c}}$} \\
\hline & $\begin{array}{l}\text { Ataxic } \\
\text { (Given Spastic) }\end{array}$ & $\begin{array}{l}\text { Spastic } \\
\text { (Given Ataxic) }\end{array}$ & $\begin{array}{l}\text { Ataxic } \\
\text { (Given Spastic) }\end{array}$ & $\begin{array}{l}\text { Spastic } \\
\text { (Given Ataxic) }\end{array}$ \\
\hline Cerebellar vermis & $-0.47^{e}$ & $-0.28^{\mathrm{d}}$ & $-0.34^{d}$ & -0.09 \\
\hline Cerebellar hemispheres & $-0.48^{\mathrm{e}}$ & $-0.36^{d}$ & $-0.41^{d}$ & -0.12 \\
\hline Brainstem & $-0.45^{e}$ & $-0.30^{\mathrm{d}}$ & $-0.42^{d}$ & -0.09 \\
\hline Thalamus & -0.14 & -0.19 & -0.05 & -0.10 \\
\hline Cerebral cortex & -0.19 & $-0.35^{d}$ & $\cdots$ & $\cdots$ \\
\hline
\end{tabular}

${ }^{\text {a }}$ Partial correlations were calculated to determine the relationship of each speech characteristic to the ICMRGlc without the contriburion of the other speech characteristic. For example, ataxic speech characteristics were correlated to lCMRGic without the interactive effects of spastic speech.

b Absolute ICMRGle in $\mathrm{mg} / 100 \mathrm{gm} / \mathrm{min}$.

'ICMRGlc normalized to the cerebral cortex.

${ }_{p} p<0.05$

e $p<0.01$.

lCMRGlc $=$ local cerebral metabolic rate for glucose.

the interactive effects responsible came from examination of ICMRGlc data normalized to the cerebral cortex. These data revealed that reduced ICMRGlc in the cerebellum and brainstem was significantly correlated with the severity of ataxia in speech and not with the severity of spasticity in speech.

Use of the partial correlations method clarified the situation. Elimination of spastic speech as a variable resulted in a significant inverse correlation between the level of ataxia in speech and absolute ICMRGlc in the cerebellum and brainstem, but not in the cerebral cortex or thalamus. Thus, the significant relationship of ataxic speech to cerebral cortical metabolic rate observed in the initial analysis appeared to result from the interactive effects of spastic speech. Furthermore, elimination of ataxic speech as a variable resulted in a significant inverse correlation between the degree of spasticity in speech and absolute ICMRGlc in the cerebellum, brainstem, and cerebral cortex. These findings were probably influenced strongly by the interactive effects of spasticity in speech and cerebral cortical metabolic rates. Indeed, after the lCMRGlc data were normalized to the cerebral cortex, the partial correlations showed that the level of ataxia in speech had a significant inverse correlation with 1CMRGlc only in the cerebellum and brainstem, and not in the thalamus or cerebral cortex. In contrast, the level of spasticity in speech showed no correlation with normalized ICMRGlc in the cerebellum, brainstem, or thalamus. We conclude from this analysis that the severity of ataxia in speech is inversely related to the level of metabolic activity in the cerebellum and brainstem and the severity of spasticity in speech is inversely related to the global level of metabolic activity in the cerebral cortex.
The findings of this study support the view that in OPCA the severity of ataxic dysarthria is related to the functional activity of the cerebellum and its connections in the brainstem. This corresponds to classical ideas concerning the central position of these structures in the pathogenesis of ataxic speech [3]. This study gives no information on the relative importance of individual component structures of the cerebellum and brainstem in speech disorders. OPCA leads to progressive degeneration of most elements of the cerebellum bilaterally, usually with no strikingly focal atrophy. In this situation, correlations between the activity of cerebellar substructures and the severity of the speech disorder cannot demonstrate the importance of any single small region. Patients with focal diseases affecting the cerebellum will need to be studied to obtain further information on the substructures important in the development of ataxic speech.

This work was supported in part by National Institutes of Health grants NS 15655 and NS 00908.

We are indebted to the staff of the Division of Nuclear Medicine for their participation in this study and to Dr Morton Brown for advice on statistical analysis of the data.

\section{References}

1. Eadie MJ. Olivo-ponto-cerebellar atrophy (Dejerine-Thomas type). In: Vinken PJ, Bruyn GW, eds. Handbook of clinical neurology. vol 21. Amsterdam: North Holland, 1975:415-431

2. Eadie MJ. Olivo-ponto-cerebellar atrophy (Menzel type). In: Vinken PJ, Bruyn GW, eds. Handbook of clinical neurology. vol 21. Amsterdam: North Holland, 1975:433-449

3. Gilman S, Bloedel JR, Lechtenberg R. Disorders of the cerebellum. Philadelphia: Davis Co, 1981

4. Harding AE. The hereditary ataxias and related disorders. London: Churchill Livingstone, 1984 
5. Gilman S, Kluin K. Perceptual analysis of speech disorders in Friedreich's disease and olivopontocerebellar atrophy. In: Bloedel JR, Dichgans J; eds. Cerebellar functions. Berlin: Springer Verlag, 1984

6. Gilman S, Markel DS, Koeppe RA, et al. Cerebellar and brainstem hypometabolism in olivopontocerebellar atrophy studied with positron emisssion tomography. Ann Neurol 1988;23:223-230

7. Darley FL, Aronson AE, Brown JR. Differential diagnostic patterns of dysarthria. J Speech Hear Res 1969;12:246-269

8. Darley FL, Aronson AE, Brown JR. Clusters of deviant speech dimensions in the dysarthrias. J Speech Hear Res 1969;12:462496

9. Darley FL, Aronson AE, Brown JR. Motor speech disorders. Philadelphia: Saunders, 1975

10. Mills CK, Weisenburg TH. Cerebellar symptoms and cerebellar localization. JAMA 1914;63:1813-1818

11. Holmes $\mathbf{G}$. The symptoms of acute cerebellar injuries due to gunshot injuries. Brain 1917;40:461-535

12. Brown JR. Localizing cerebellar syndromes. JAMA 1949;141: 518-521

13. Dow RS, Moruzzi G. The physiology and pathology of the cerebellum. Minneapolis: University of Minnesota Press, 1958
14. Amici R, Avanzini G, Pacini L. Cerebellar tumors. Monographs in neural sciences. vol 4. Basel: Karger, 1976

15. Lechtenberg R, Gilman S. Speech disorders in cerebellar disease. Ann Neurol 1978;3:285-290

16. Kluin KJ, Gilman S, Markel D, et al. Speech disorders in olivopontocerebellar atrophy correlate with regional abnormalities of cerebral metabolic activity studied with PET. Neurology 1987;37(suppl 1):268

17. Ehrenkaufer RE, Potocki JF, Jewett DM. Simple synthesis of F18-labeled 2-fluoro-2-deoxy-D-glucose: concise communication J Nucl Med 1984;25:333-337

18. Phelps ME, Huang SC, Hoffman EJ, et al. Tomographic measurement of local cerebral glucose metabolic rate in humans with (F-18) 2-fluoro-2-deoxy-D-glucose: validation of method. Ann Neurol 1979;6:371-388

19. Hawkins RA, Mazziotta JC, Phelps ME, et al. Cerebral glucose metabolism as a function of age in man: influence of the rate constants in the fluorodeoxyglucose methods. J Cereb Blood Flow Metab 1983;3:250-253

20. Gilman S, Markel DS, Koeppe RA, et al. A comparison of CT and PET findings in olivopontocerebellar atrophy. Ann Neurol 1986;20: 121 